\title{
MEASUREMENT OF PAIR-PRODUCTION BY HIGH ENERGY PHOTONS IN AN ALIGNED TUNGSTEN CRYSTAL
}

\author{
R.Moore, M.A.Parker \\ Cavendish Laboratory, Cambridge University, UK
}

A.Baurichter, K.Kirsebom, R.Medenwaldt, U.Mikkelsen, S.P.Møller, E.Uggerhøj, T.Worm

Institute for Synchrotron Radiation, Aarhus University, Aarhus, Denmark

N.Doble, K.Elsener

European Organization for Nuclear Research (CERN), Geneva, Switzerland

S.Ballestrero, P.Sona

University of Firenze and INFN, Firenze, Italy

V.M. Strakhovenko

Novosibirsk, Russia

C. Biino ${ }^{*}$

University of Torino and INFN, Torino, Italy

Z.Z.Vilakazi

University of Witwatersrand, Johannesburg, South Africa

\begin{abstract}
A new measurement has been made of the rate of pair-production in a $3.2 \mathrm{~mm}$ thick tungsten crystal, exposed to photons with energies in the range 10 to $150 \mathrm{GeV}$, for angles of incidence up to $10 \mathrm{mrad}$ from the crystal axis. A strong enhancement of the pair-production rate is observed when the beam is aligned along the $\langle 100\rangle$ crystal axis, as compared to a random orientation. This effect can be exploited in the NA48 $\mathrm{CP}$-violation experiment by using a thin crystal rather than an amorphous material to convert photons, thus minimising the scattering of kaons in the converter.
\end{abstract}

Presented at the Workshop on 'Channeling and other Coherent Crystal Effects at Relativistic Energies', Aarhus, Denmark, July 10-14, 1995

Submitted to Nuclear Instruments and Methods B

Geneva, Switzerland

July 1995

*) Present address, SL Division, CERN, Geneva, Switzerland 
The dominant absorption process for high-energy photons in matter is pair production. A photon may turn into an electron/positron pair in the electromagnetic field of the target nuclei and electrons. The presence of an external field assures energy and momentum conservation. The cross-section is given by the Bethe-Heitler value characterised by the energy-independent radiation length of the material.

When photons are incident onto a crystal at a small angle to a crystallographic axis or plane, the pair production cross-section may be enhanced over the value pertaining to an amorphous target. The coherent interaction with the atoms lying in strings or planes gives rise to this enhancement. This is the so-called 'coherent pair production' developed in the fifties and reviewed in [1].

The strong field required for photon conversion only exists near atomic nuclei and electrons, and although of very large magnitude it only extends over distances of the order of one Angstrom. When the photon direction is at a very small angle to a string of atoms, the field from the individual atoms may in some cases be replaced by an average field obtained by smearing the atomic charges along the string. In this continuum approximation, describing the channeling of charged particles through crystals [2], a photon incident at a very small angle to the atomic string experiences a strong field $\left(10^{9}\right.$ to $\left.10^{10} \mathrm{~V} / \mathrm{m}\right)$ which is macroscopic along the string direction. This leads to an even greater enhancement than for coherent pair production, which can be calculated as taking place in a constant field. The point is that the field from the string of atoms can be considered constant over the pair-creation formation length. Whereas the coherent pair production can be calculated in the Born limit, the constant field pair production has to be calculated exactly using quantum electrodynamics. Details of these calculations can be found in [3]. The characteristic angle separating the regions of constant-field and coherent pair-production is given by $V_{0} / m c^{2}$ where $V_{0}$ is the depth of the continuum potential and $m c^{2}$ is the electron rest energy. A kinematic threshold for this effect occurs when the photon energy is equal to $2\left(m c^{2}\right)^{2} / V_{0}$, but considerably higher energies are required to obtain a rate equal to the Bethe-Heitler cross-section. This threshold has been estimated in [3] and [4], and is given by $m^{3} c^{4} \rho d / \hbar^{2} \mathrm{Z} \alpha$. Here $\rho$ is the thermal vibration amplitude of the atoms, $\mathrm{d}$ the atomic spacing, $\mathrm{Z}$ the atomic number and $\alpha$ the fine structure constant. This threshold is around 120, 50 and $20 \mathrm{GeV}$ for silicon, germanium and tungsten respectively, but the lower threshold for high-Z materials is accompanied by a lower maximum enhancement $($ see $[3,4])$.

Previous measurements on constant-field pair production have only been made on germanium in the energy range 50 to $150 \mathrm{GeV}[5,6]$, and the results have been in good agreement with calculations. Some measurements of early shower development in tungsten have also been made at energies of $8-27 \mathrm{GeV}[7]$.

This effect could find an experimental application in the NA48 CP-violation experiment at CERN [8]. A detector placed in the $\mathrm{K}_{\mathrm{S}}$ beam will observe photons from $\mathrm{K}_{\mathrm{S}} \rightarrow \pi^{0} \pi^{0} \rightarrow 4 \gamma$ decays and define the upstream boundary of the decay fiducial region. Photons must be detected by inducing pair-production in a converter, but neutral kaons passing through the converter should be disturbed as little as possible. An aligned crystal used as a converter could be made with reduced thickness and still maintain the same conversion efficiency, whilst reducing the average scattering angle of the kaons. A material with as low a threshold as possible is required. Crystals must be obtained with a mosaic spread less than the characteristic angle $V_{0} / m c^{2}$. Tungsten is therefore a suitable choice.

In this paper measurements of the pair-production enhancement in tungsten are 


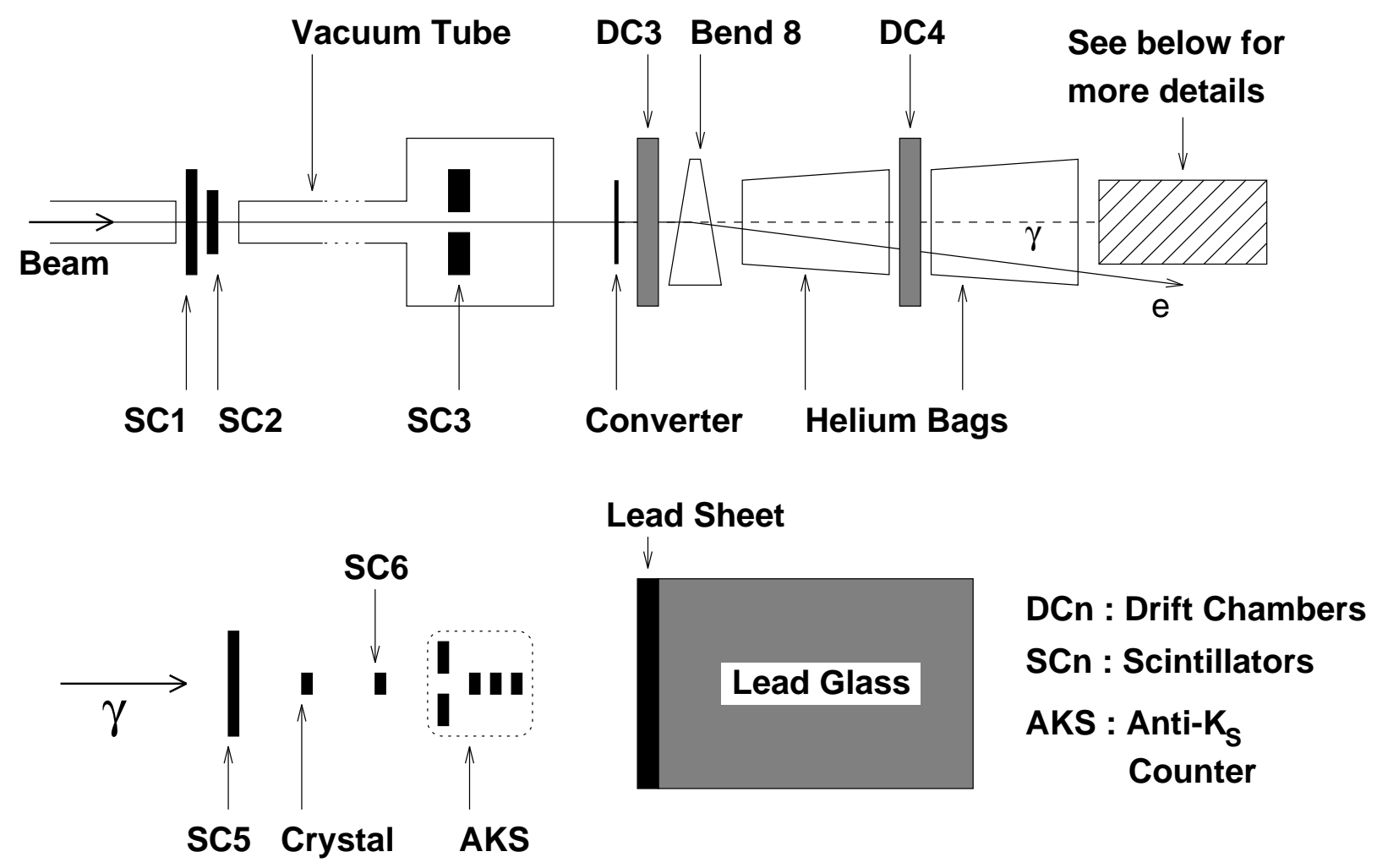

Figure 1: The layout of the apparatus in the H2 beamline

presented with photon energies between 10 and $150 \mathrm{GeV}$ in order to study the energy dependence, and at a variety of incident angles, up to $10 \mathrm{mrad}$ from the $\langle 100\rangle$ axis. The enhancement $(\eta)$ is defined as the ratio of the radiation length for an amorphous material to that of the crystalline material, corresponding to an increase in the photon pair-production cross-section.

\section{The Experimental Apparatus}

The measurements were made by counting the number of converted and unconverted photons emerging from a tagged photon beam incident onto an aligned tungsten crystal.

The experimental apparatus was set up in the H2 secondary beam line at the CERN SPS. The layout used is shown in Figure 1. The electron beam, the energy of which could be varied up to a maximum of $150 \mathrm{GeV}$, had a divergence of $\pm 30 \mu \mathrm{rad}$, and a spot size of $1.4 \mathrm{~cm}$ diameter was selected by the trigger scintillation counters (SC1, SC2 and SC3). The electron beam trigger used for this analysis required signals from SC1 and SC2, and no signal from SC3 (a square scintillation counter with a hole defining the beam acceptance). The beam was incident onto a radiator, consisting of a $1 \mathrm{~mm}$ thick sheet of copper (7\% of a radiation length) to induce bremsstrahlung. The outgoing electron was deflected away from the beam axis using a $1.77 \mathrm{Tm}$ magnet. Its position before and after the magnet was measured with a precision of $100 \mu \mathrm{m}$ in orthogonal coordinates by a pair of drift chambers (DC3 and DC4). These measurements allowed the energy of the bremsstrahlung photon to be calculated with a precision higher than that available from the lead glass array, for photons up to $100 \mathrm{GeV}$ in energy. Beyond this, the deflection of the electron was such that the track lay outside of the acceptance of DC4. Upstream and downstream of DC4, helium bags were placed to reduce the probability of the photon 
interacting before reaching the crystal.

The crystal was placed on a goniometer with a scintillation counter (SC5) immediately upstream. This scintillation counter allowed photons which produced showers upstream of the crystal to be vetoed. Downstream of the crystal, a scintillation counter (SC6) was used online to monitor showering in the crystal. In addition, the NA48 'Anti decayed $\mathrm{K}_{\mathrm{S}}$ ' (AKS) counter was placed after the crystal to measure the showers produced. The AKS counter will be used in NA48 to veto both charged and neutral decays in the

$\mathrm{K}_{\mathrm{S}}$ beam, to define the beginning of the decay region, and to calibrate the electromagnetic calorimeter. It consists of 4 layers of scintillation counters. The first layer is larger with a central hole while the other 3 layers cover the beam axis. Further details of the construction and use of this counter can be found in [9]. Finally, the photon energy was measured using 4 blocks of lead glass in a square array. A lead sheet, 2 radiation lengths thick, was placed at the front of the blocks. Each block was $7.5 \mathrm{~cm}$ square and $36 \mathrm{~cm}$ long in the beam direction. The array was read out using a single photomultiplier tube directly connected to all the blocks. The lead glass array allowed photon energies to be measured beyond the $100 \mathrm{GeV}$ limit imposed by the drift chamber acceptance.

The tungsten crystal had an irregular shape cut from a rod at $45^{\circ}$ with an average thickness of $3.17 \mathrm{~mm}$ in its central part. The mosaic spread of the crystal was $\sim 300 \mu \mathrm{rad}$ full width at half maximum. It was supplied with a mirror fixed to its surface perpendicular to the $\langle 100\rangle$ axis. Figure 4 shows the crystal mounted in its support. The mirror can be seen as a white dot on the surface. A laser placed on the beam axis was used to make a pre-alignment of the crystal by obtaining reflection at normal incidence. The crystal was aligned by measuring the rate of showers in the crystal, giving signals greater than 1 m.i.p. in SC6, using a direct electron beam, with the converter plate removed from the beam line. Showering from incident electrons is also expected to be enhanced when the beam is incident along a crystal axis. Figure 2 shows the relative counting rate seen as the crystal was rotated by $\pm 20 \mathrm{mrad}$ such that the incident beam was in a plane $17 \mathrm{mrad}$ from the $\langle 100\rangle$ axis. A broad enhancement of the intensity distribution, with a FWHM of $15 \mathrm{mrad}$, is seen as the $<100\rangle$ axis passes close to the beam direction. Superimposed on this are narrow peaks at angular positions where the beam direction coincides with the directions of the $(100)$ weak crystal plane and the (110) strong crystal planes. The orientation of these planes and the direction of the scan of the beam are shown in Figure 3.

The photon beam was used to check the positioning of the crystal in the beam. The positions of electrons were measured in DC3 before bremsstrahlung occured. Events were selected in which a shower was observed downstream of the crystal in the conversion scintillation counter SC6. The fraction of photons which convert is shown as a contour plot as a function of the position of the parent electron in Figure 5. The shape of the crystal is clearly seen. The variation in the number of showers seen is due to the variation in thickness of the crystal near its edges and the small support ring upon which it was mounted. Events in the good region of the crystal were selected using the triangular cut shown on the figure.

\section{$3 \quad$ Measurement of the photon energies}

The lead glass array was used to measure the photon energies in order to provide a uniform measurement up to the highest energy available. An initial calibration was made using direct electrons of $149.1 \mathrm{GeV}$ which did not pass through the crystal. The energy detected was measured with a photomultiplier tube and ADC. A point at zero energy was 


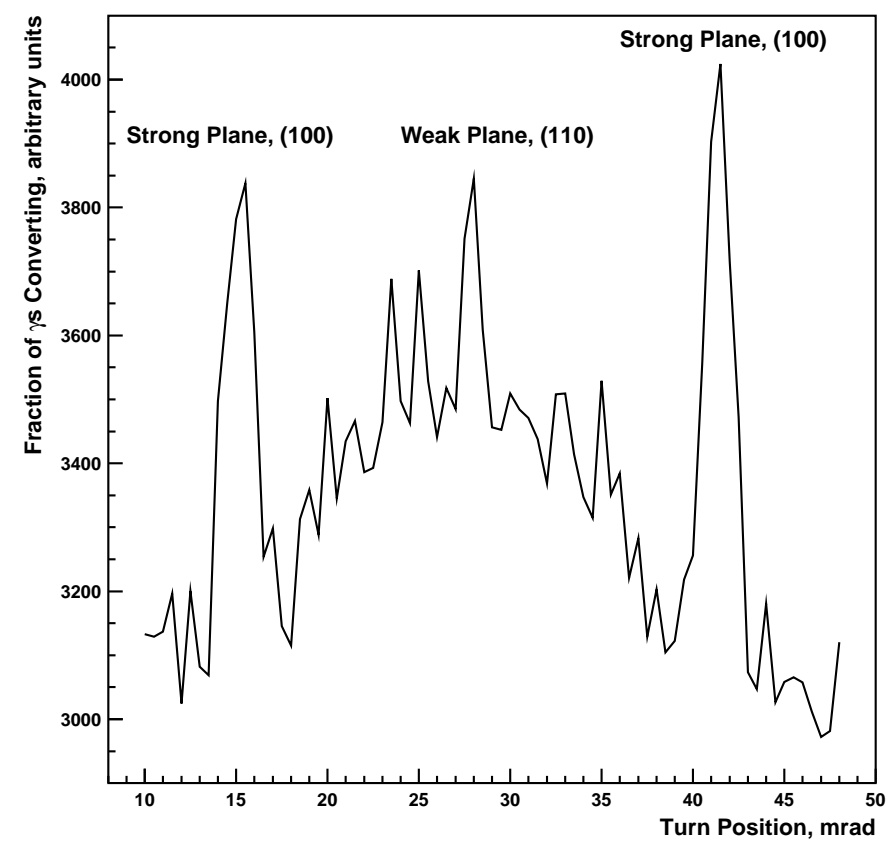

Figure 2: Alignment of the crystal axis using an electron beam. The rate of electron induced showers is plotted as the crystal is rotated (see text).

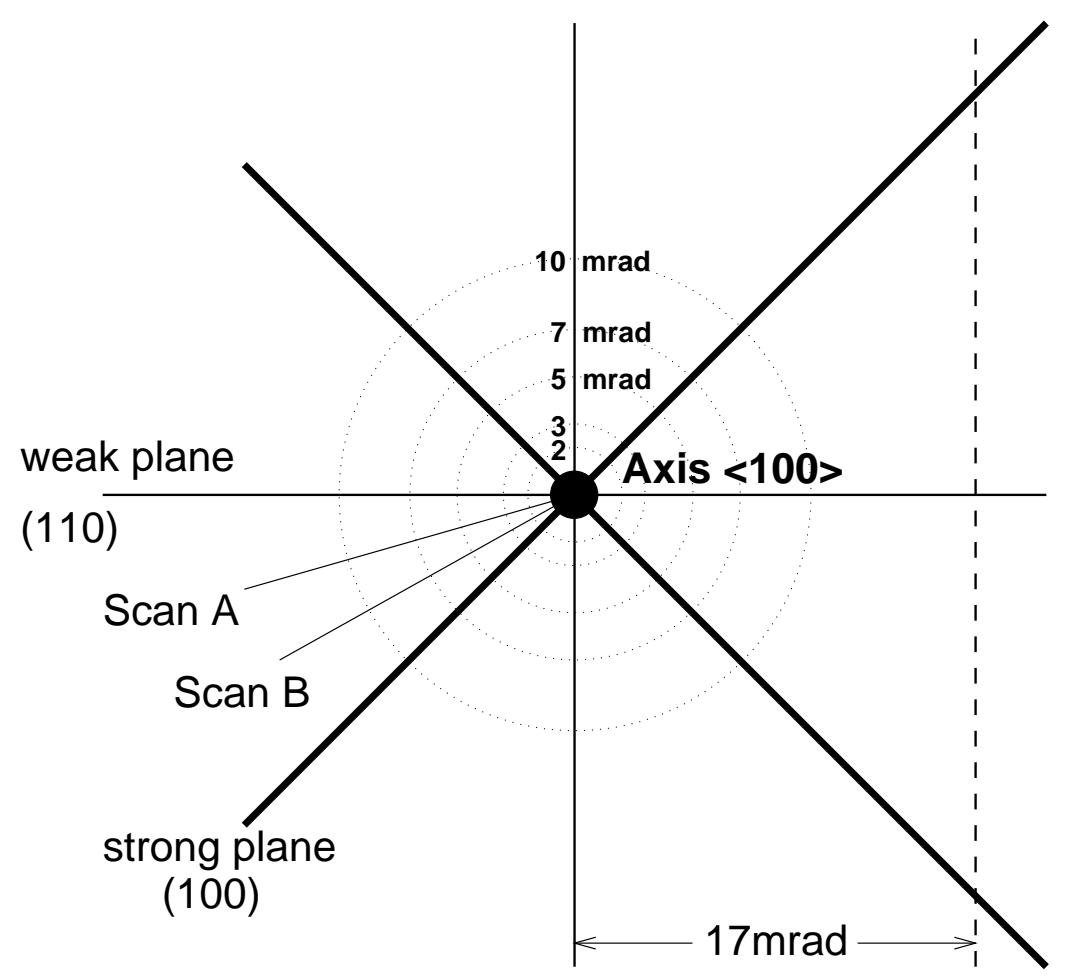

Figure 3: Orientation of the strong and weak planes of the tungsten crystal with respect to the crystal axis. The plane scanned by the electron beam used for alignment is shown by the dashed line, The photon beam used in the conversion analysis was scanned along the strong and weak planes, and scans $\mathrm{A}$ and $\mathrm{B}$. 


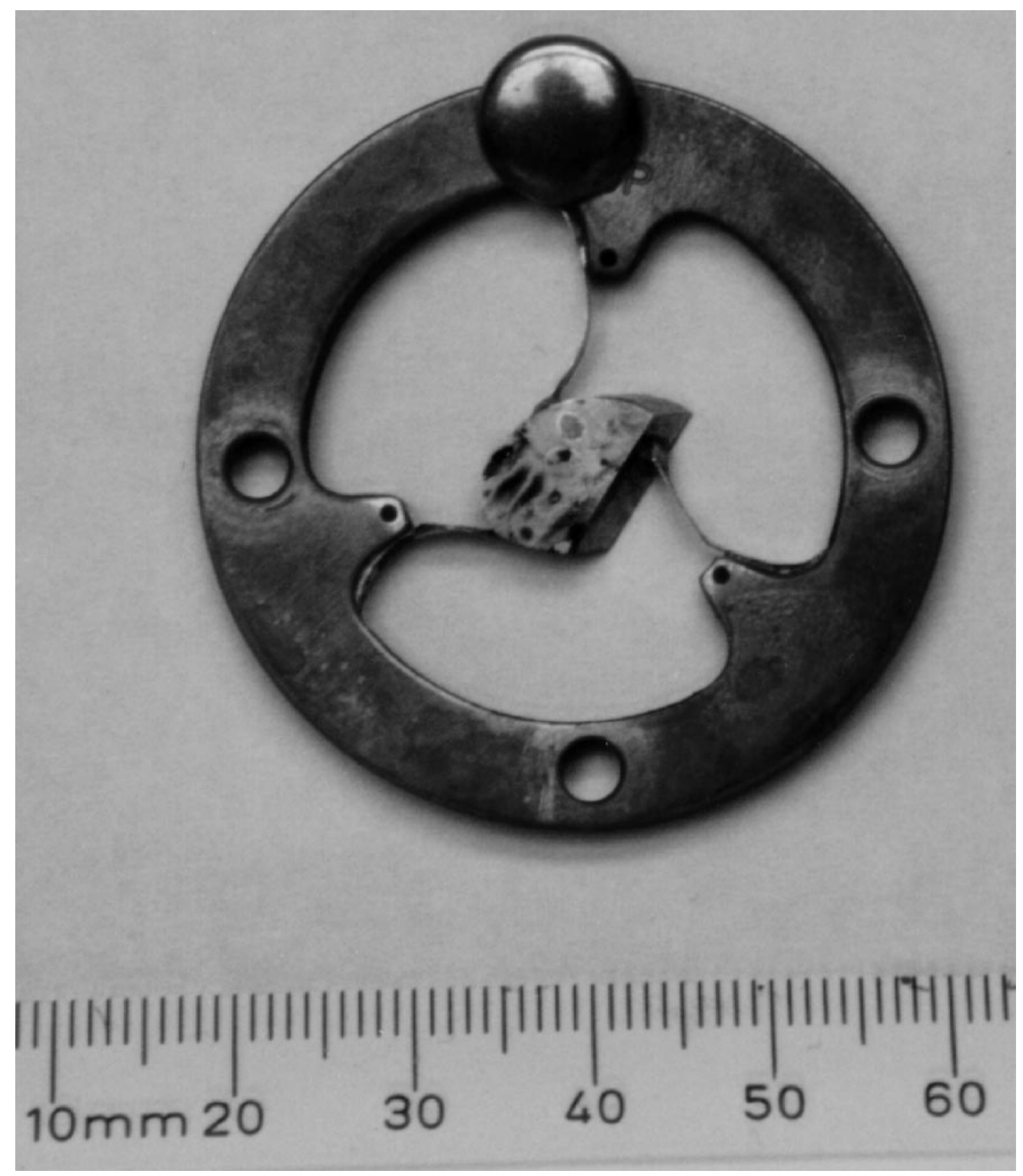

Figure 4: Photograph of the tungsten crystal on its support ring. The grey circle on the crystal surface is the mirror used for pre-alignment. 




Distance in $\mathrm{x}, \mathrm{cm}$ 